\title{
Application of small intestine decompression combined with oral feeding in middle and late period of malignant small bowel obstruction
}

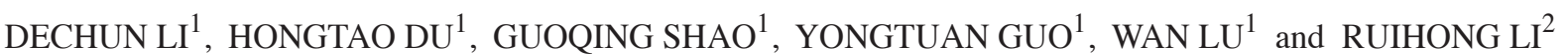 \\ ${ }^{1}$ Department of Radiology, Xuzhou Affiliated Hospital of Southeast University; \\ ${ }^{2}$ Department of Nutriology, Xuzhou Central Hospital, Xuzhou, Jiangsu 221009, P.R. China
}

Received March 28, 2016; Accepted September 20, 2016

DOI: $10.3892 / \mathrm{ol} .2017 .6153$

\begin{abstract}
The application value of small intestine decompression combined with oral feeding in the middle and late period of malignant small bowel obstruction was examined. A total of 22 patients with advanced malignant small bowel obstruction were included in the present study. An ileus tube was inserted via the nose under fluoroscopy into the obstructed small intestine of each patient. At the same time, the insertion depth the of the catheter was adjusted. When the catheter was blocked, small bowel selective angiography was performed to determine the location and cause of the obstruction and the extent of the obstruction, and to determine the length of the small intestine in the site of obstruction, and to select the variety and tolerance of enteral nutrition. We observed the decompression tube flow and ease of intestinal obstruction. In total, 20 patients were treated with oral enteral nutrition after abdominal distension, and 22 cases were treated by the nose to observe the drainage and the relief of intestinal obstruction. The distal end of the catheter was placed in a predetermined position. The symptoms of intestinal obstruction were relieved 1-4 days after decompression. The 22 patients with selective angiography of the small intestine showed positive X-ray signs: 18 patients with oral enteral nutrition therapy had improved the nutritional situation 2 weeks later. In 12 cases, where there was anal defecation exhaust, 2 had transient removal of intestinal obstruction catheter. In conclusion, this comprehensive treatment based on small intestine decompression combined with enteral nutrition is expected to become a new therapeutic approach and method for the treatment of patients with advanced tumor small bowel obstruction.
\end{abstract}

Correspondence to: Dr Ruihong Li, Department of Nutriology, Xuzhou Central Hospital, 199 South Jiefang Road, Xuzhou, Jiangsu 221009, P.R. China

E-mail: lidechun7878@sina.com

Key words: malignant, small bowel obstruction, enteral nutrition, intestine decompression

\section{Introduction}

Intestinal obstruction caused by malignant tumors is a common complication of late cancer, and is especially common in patients with gastrointestinal malignant cancer and gynecologic malignant cancer $(1,2)$. The small intestine has a relatively concentrated distribution range, making it easy to adhere after abdominal surgery. It has abundant blood supply and is thus vulnerable to tumor metastasis in the peritoneal cavity and development of intestinal canal stenosis and compression which causes small intestinal obstruction (2). These patients are typically at a late stage of cancer, and mostly are unable to undergo surgery. The traditional treatment method is feeding via the nasogastric tube. This method cannot thoroughly drain to the small intestine proximal end, thus its effect on small intestine distal end drainage is not that effective.

In the present study, we used nasotracheal small intestine decompression tubes to perform small intestine decompression drainage on this population of patients, which can relieve symptoms of intestinal obstruction in the short-term. Subsequently, we used decompression tubes for selective opacification of the distal end of the small intestine to assist the diagnosis, and carried out enteral nutrition treatment and achieved positive treatment effects.

\section{Materials and methods}

Patients. In total, 22 cases of middle and late stage malignant tumor causing-secondary small intestine obstruction by nasogastric small intestine decompression were treated. Of the 22 cases, there were 12 males and 10 females, aged 28-81 years, median age 49 years. A total of 16 cases had a history of surgery related to malignant tumors ( 9 cases of stomach cancer, 1 case of porta hepatis bile duct cancer, 1 case of hepatocarcinoma, 4 cases of ovarian cancer, and 1 case of cervical cancer), 6 cases had primary lesions and secondary peritoneal metastasis ( 3 cases of stomach cancer, 1 case of liver cancer, 1 case of pancreatic cancer and 1 case of cervical cancer), and 4 cases had a history of radiotherapy. All the patients were diagnosed with small intestine obstruction by clinical diagnosis, as well as plain film and CT of the abdomen. A total of 16 cases were clinically considered 
intestinal obstruction by abdominal adhesion and 6 cases were of intestinal obstruction caused by tumor compression and invasion. The patients showed symptoms including stomach and abdominal distension, emesis, and inability to defecate and pass flatus. This study was approved by the Ethics Committee of Xuzhou Central Hospital. Signed written informed consents were obtained from all participants before the study.

Tube array method and small intestine decompression. A nasogastric decompression tube (with 2 cavities, 2 air sacs and multiple lateral drainage apertures on the anterior of the tube) was used. The tube was placed through the nostril, stomach and duodenum to the upper jejunum (distal of Treitz ligament) with X-ray monitoring and assistance by a metal guidewire. For patients with significant structural changes to the upper digestive tract or difficulty in inserting the tube after surgery, we used a gastroscope to assist the tube in reaching the jejunum, and inserted the tube into the distal end of the small intestine as far as possible with the combination of the tube and a guidewire. When tube insertion was hindered, we injected $100 \mathrm{ml}$ lohexol by the decompression tube and evaluated the extent of small intestine expansion. We injected $5-15 \mathrm{ml}$ sterilized distilled water to the anterior air sacs, and the water-filled sacculus stimulated the alimentary canal to spur the tube to gradually move to the distal end of the small intestine. The suction socket of the tube was connected to negative pressure drainage. The tube was fixed to the nose by adhesive tape to prevent the decompression tube from sliding out of the body. Each day, patients were supervised and tube position adjusted, and observed for improvements in the condition of gas-liquid levels within the enteric cavity, whether there was free gas under the diaphragm, and the position of previously injected contrast medium in the intestine. We inserted the guidewire again to adjust the tube when necessary. Each day the amount of tubal drainage was recorded, and we carried out regular microorganism tests for the first drainage, and supervised the changes in body weight and biochemistry of blood each week.

Selective small intestine opacification diagnosis. After $24 \mathrm{~h}$, if the tube did not naturally continue to move deeper, or if it still could not move forward by adjustments, then we immediately injected contrast medium through the decompression tube mouth to carry out selective small intestine opacification. When there was opacification, the anterior sacculus was placed under pressure and we used $20-30 \mathrm{ml}$ gas to fill the posterior sacculus to let it tightly bind to the intestinal wall, and to prevent the reflux of contrast medium. When opacification occurred, we mainly observed the extent of intestinal tract expansion, unobstruction, passage and stenosis. When the opacification was finished, the posterior sacculus was placed under pressure and we filled the anterior sacculus with $5 \mathrm{ml}$ sterilized distillated water. We then continued the decompression drainage (3).

Enteral nutrition treatment. At $48 \mathrm{~h}$ after the disappearance of abdominal distension, felt by the patient him/herself, and under the circumstance of clear clinical diagnosis that the patients did not have complications such as perforation of the intestine and intestinal necrosis, we immediately began enteral nutrition treatment. For patients with selective small intestinal opacification showing unobstructed intestine, we injected 200-500 $\mathrm{ml}$ saline to the small intestine through the decompression tube $(100 \mathrm{ml} / \mathrm{h})$, and turned off the drainage pathway and observed for 3-4 h. If there was no obvious discomfort, then we intermittently turned off the drainage pathway and changed to oral administration of short peptides and glutamine. We gradually increased the amount. When the total amount reached $>800 \mathrm{ml}$ per day, we gradually increased nutrition with small amounts of chyme until normal diet was achieved, and the patients could remove the tube. During this period, close observation of abdominal symptoms and signs were still needed, and when abdominal distension or abdominal pain appeared when taking food, we immediately turned on the drainage tube to carry out decompression drainage. For patients with intestinal obstruction and with obvious stenosis, distortion or obstruction detected by selective small intestine opacification, and for where the calculated length of small intestinal canal upper obstruction was $>1 \mathrm{~m}$, we gave oral saline $(100 \mathrm{ml})$, and if abdominal distension did not significantly increase, then we began oral administration of short peptides without glutamine. We then gradually increase the amount, meanwhile continuously carrying out negative pressure drainage by the decompression tube. Approximately 1 week later, we re-evaluated small intestine opacification to observe the extent of intestinal canal expansion and improvement from stenosis. The lacking part of dietary caloric intake was accompanied by parenteral nutrition.

\section{Results}

Tube array and treatment. A total of 21 cases of patients were successful in terms of insertion of the nasogastric decompression tube to the jejunum position on the first attempt, with the operation time lasting 12-86 min, averaging $29 \mathrm{~min}$. For 1 case, insertion of the decompression tube was successful 1 day after stomach intestinal decompression. After $10 \mathrm{~h}$ to 1 week from tube decompression (average $~ 2.2$ days), all patients had lessened symptoms of abdominal distension, abdominal pain and vomiting, and tube drainage liquid volume was 350-3,650 $\mathrm{ml}$ (average 1,280 ml) per day within 1 week. After 1 week of decompression, plain film of the abdomen (in decubitus position) showed that 17 patients had lost liquid-gas levels in the intestinal cavity, and did not have obvious intestine expansion, with insertion depth 1.6-2.9 m (average $\sim 2.2 \mathrm{~m}$ ). Among the 17 patients, 12 patients had the ability to exhaust and a small amount of excrement to exit the body. The 17 patients were prescribed special enteral nutrition through the mouth with the tube, and 2 patients underwent surgical small intestine stoma treatment and we removed the tubes after 5 weeks when their symptoms related to intestinal obstruction abated. As for the other patients, 5 had lessened symptoms related to intestinal obstruction but still had air-fluid level at the distal intestinal tube, 4 patients were not suitable for enteral nutrition since the obstruction position was $\sim 1 \mathrm{~m}$ from the jejunum, 1 patient carried out the special-prescription enteral nutrition through tube feeding but insufficient nutrition was supported by parenteral nutrition. None of the patients had complications related to the operation such as alimentary tract hemorrhage or piercing. 
Selective small intestine opacification. For all the 22 patients, the anterior part of the tube stopped moving forward to the deep part of small intestine after several adjustments, and after $24 \mathrm{~h}$, contrast medium was injected through the tube to carry out intestinal opacification. Opacification of 11 patients showed that the small intestine canal was obstructed at the anterior of the decompression end, as contrast medium cannot pass the obstructed segment of the intestinal canal, and the proximal intestinal canal expands (Fig. 1). Opacification of the 8 patients showed that contrast medium slowly enters the intestinal canal at the distal tube, but it cannot clearly show the condition of a narrowed intestinal canal segment because of the overlap of the intestinal canal (Fig. 2), among which 3 patients had expansion of the distal intestinal canal (Fig. 3). Opacification of the 3 patients showed that the small intestine at the distal end had localized intestinal canal stenosis and the border was irregular, which was considered a result of tumor invasion (Fig. 4). Of the 22 patients, 4 were able to receive enteral nutrition because the position of the obstruction was $\sim 1 \mathrm{~m}$ away from the jejunum.

Application of small intestine decompression combined with eating through the mouth. Among the 22 patients, 18 patients underwent enteral nutrition at the same time as small intestine decompression, of which 6 patients had moderate abdominal distension at the beginning of oral enteral nutrition and expressed tolerance after proper adjustments of both the time and amount. A total of 18 patients did not show abdominal distension, abdominal pain or signs of peritoneal activation during the treatment period of eating, and had increased serum albumin and prealbumin without significant weight loss after 2 weeks of treatment (Table I).

\section{Discussion}

The increase of tumor incidence year by year leads to increased incidence of malignant intestinal obstructions. For malignant intestinal obstructions whereby the obstruction and origin cannot be removed by surgery, emesis, abdominal pain and abdominal distension occurs in patients, while they are afflicted with the incapacity to eat. Presently, the principles and prescription of nutrition for patients with such tumors remains the common means of nutritional support for patients with common or more serious gastrointestinal problems. Patients with gastrointestinal function prefer enteral nutrition, while patients with gastrointestinal function insufficiency or disability prefer parenteral nutrition $(4,5)$. It is recommended to combine enteral nutrition and parenteral nutrition. Maintainance of the intestinal endometrium barrier and immune function occurs by enteral nutrition, while parenteral nutrition provides energy and nutritional substrates. Small intestinal obstruction is a common complication in patients with middle and late stage malignant tumors, and may endanger life if not treated in time. In treatment, not only is the prompt removal of intestinal obstruction needed, but consideration must be given to diagnosis of the cause of the obstruction and a nutrition support plan, to give advantage to later treatment. Mortality 30 days after late period tumor intestinal obstruction surgery is $\sim 9-40 \%$, and the complication incidence is $99.0 \%$. Therefore, late stage tumor causing

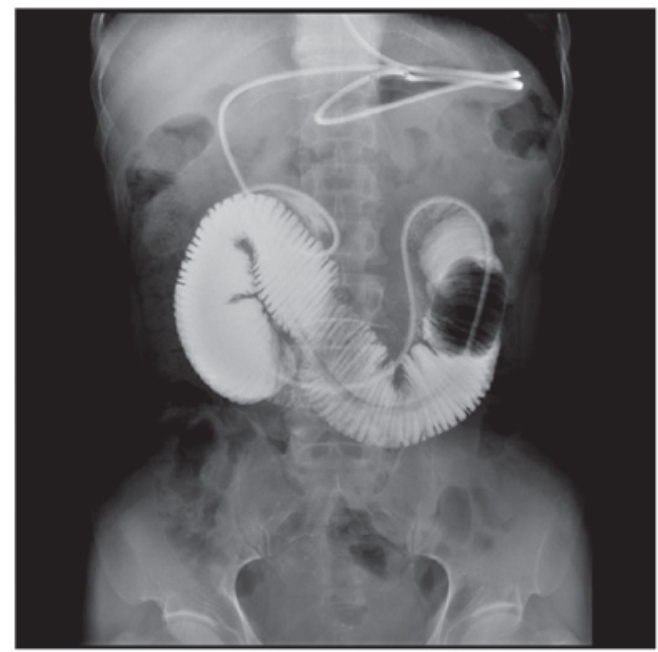

Figure 1. Small intestinal canal obstruction of decompression tube anterior, contrast medium cannot pass obstructed segment of intestinal canal, and proximal intestinal canal expands.

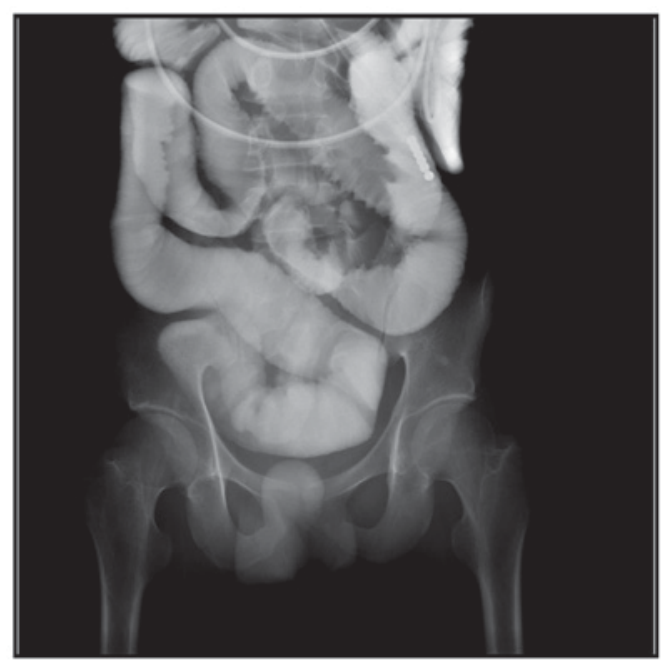

Figure 2. Contrast medium slowly enters distal end of tube in intestinal canal, but due to the overlap of the canal, stenosis of the intestinal segment cannot be showed clearly.

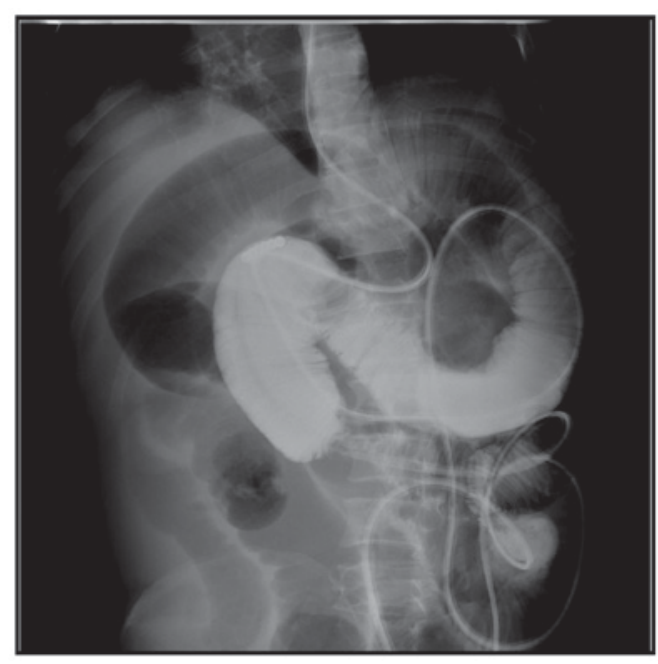

Figure 3. Small intestine at the distal end of the tube has localized stenosis, and there is expansion at distal end of the stenosed segment. 
Table I. Nutrition index before and after enteral nutrition.

\begin{tabular}{lccc}
\hline Characteristics & $\begin{array}{c}\text { Albumin, } \\
\mathrm{g} / \mathrm{l}\end{array}$ & $\begin{array}{c}\text { Prealbumin, } \\
\mathrm{mg} / \mathrm{l}\end{array}$ & $\begin{array}{c}\text { Body } \\
\text { weight, } \mathrm{kg}\end{array}$ \\
\hline Before treatment & $32.0 \pm 2.7$ & $34.1 \pm 2.3$ & $61.2 \pm 2.4$ \\
After treatment & $238 \pm 27.4$ & $316.0 \pm 28.9$ & $60.8 \pm 5.3$ \\
P-value & $\mathrm{P}<0.05$ & $\mathrm{P}<0.05$ & $\mathrm{P}<0.05$ \\
\hline
\end{tabular}

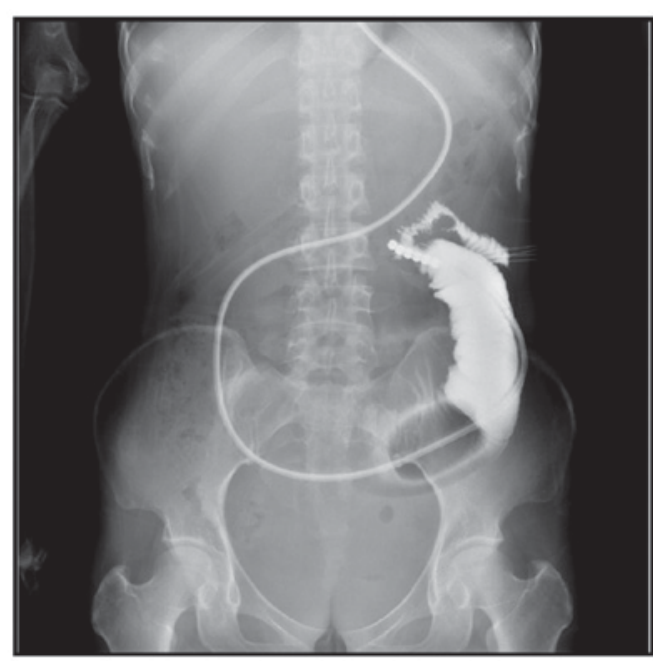

Figure 4. Opacification shows that the small intestine at the distal end of the tube has localized stenosis, and the boarder is irregular, which is considered tumor invasion.

mechanical intestinal obstruction surgery can only benefit certain patients, including those with mechanical intestinal obstruction caused by fibrosis adhesion, localized tumor solitary obstruction and chemotherapy insensitive tumor bearing patients, thus it is not appropriate to commonly choose surgical treatment.

After the occurrence of malignant small intestinal obstruction, a series of pathological and physiological changes appear, partially in the intestine and throughout the body. The most important pathophysiological consequences are edema of the intestinal dissepiment and localized tumor, and the damage caused by liquid secretion-absorption equilibrium in the intestinal canal. Localized intestinal canal stenosis causes continuous uncoordinated peristalsis and thus aggravates the intestine by expansion of the proximal end of the obstruction, and leads to an increase of intestinal cavity internal pressure, which results in intestinal mucous membrane ischemia, anoxia and intestinal wall blood transportation disability, and finally leads to intestinal wall necrosis and piercing. Hence the key to treatment is to reduce internal pressure of the intestinal cavity and efficiently drain intestinal contents, to improve intestinal wall blood transportation. Traditional nasogastric tube decompression can only decompress and drain the gastral cavity, and has poor effects on small intestine content drainage, thus is cannot reach the requirement of clinical treatment. We inserted 3-m small intestine decompression tubes to expand the small intestine and carry out whole range decompression and drainage, to treat intestinal obstruction (6). We also injected contrast medium through the decompression tube to the small intestine to carry out selective small intestinal opacification, which can assist the diagnosis and treatment.

All 22 patients in the group could drain gas and liquid (average 1,280 ml) from the small intestine per day after insertion of the nasogastric decompression tube. After $10 \mathrm{~h}$ to 1 week (average $\sim 2.2$ days) of tube decompression, all patients were relieved of their symptoms including abdominal pain, abdominal distension and vomiting. This demonstrates the effectiveness of using nasogastric decompression tubes to treat small intestinal obstruction. Small intestine opacification all showed positive results (11 patients showed that small intestine canal is obstructed at the anterior of decompression end, contrast medium cannot pass the obstructed intestinal canal segment, and the proximal intestinal canal expands. Opacification of 8 patients showed that contrast medium slowly entered the intestinal canal at the distal tube, among which 3 patients still had an expanded distal intestinal canal. Opacification of 3 patients showed that the small intestine at distal tube had localized stenosis and the border was irregular. These results are all significantly improved over regular nasogastric tube drainage. There are also multiple studies reporting the superiority of decompression tubes in aspects of decompression and drainage $(7,8)$.

Traditionally, enteral nutrition treatment is only started after full recovery of gastrointestinal function, that is the ability to pass flatus. Before the full recovery of gastrointestinal function, complete fasting is needed and patients are given total parenteral alimentation. However, an obvious weakness of this approach is that long fasting periods can cause intestinal mucous membrane atrophy, damage of barrier function, displacement of bacteria, and requires expensive long-term use of parenteral nutrition. Therefore, it is worthy of attention to carry out predigested short peptide enteral nutrition under the protection of small intestine compression and drainage, using anterior intestinal canal segment, having absorption and digestion function (9). Under the circumstance of having drainage tubes in the small intestine, 18 patients in the group had increased serum albumin, prealbumin without significant reduction of body weight after orally taking predigested short peptide nutrition, highlighting the value of eating and drawing at the same time when suffering from middle and late neoplasm related small intestinal obstructions. According to our experience, we consider patients clearly diagnosed with malignant neoplasm induced intestinal obstruction and accompanied by the following conditions are suitable for treatment with eating and drawing at the same time: i) Verified widespread metastasis in the abdominal cavity by iconography check, ii) magnanimous ascites that recurs after drainage, iii) open necrosectomy and certified that further surgery cannot be carried out, iv) previous abdominal surgery demonstrating widespread metastasis, v) widespread abdominal contents, vi) uncontrolled symptoms caused by outer abdominal cavity metastasis, vii). previous abdominal cavity or cavitas pelvis radiotherapy, and viii) poor common condition and advanced age.

To summarize, the comprehensive treatment based on small intestine decompression combined with enteral nutrition is worthy of clinical application. 


\section{Acknowledgements}

This study was supported by the Science and Technology Bureau topic (Xuzhou, Jiangsu, China), no. XZZD1353.

\section{References}

1. Beltran MA and Cruces KS: Primary tumors of jejunum and ileum as a cause of intestinal obstruction: A case control study. Int J Surg 5: 183-191, 2007.

2. Roeland $\mathrm{E}$ and von Gunten CF: Current concepts in malignant bowel obstruction management. Curr Oncol Rep 11: 298-303, 2009.

3. Abdulzhalilov MK: Ways of raising efficacy of nasointestinal drainage in patients with intestinal obstruction and general peritonitis. Khirurgiia (Mosk) 4: 39-41, 2003 (In Russian).

4. Eren T, Bayraktar B, Celik Y, Boluk S and Adali G: Acute malignant intestinal obstruction accompanied by synchronous multifocal intestinal cancer in Peutz-Jeghers syndrome: Report of a case. Surg Today 42: 1125-1129, 2012.
5. Li J: Rationality of the preference to enteral nutrition. J Parenter Enteral Nutr 20: 321-322, 2013.

6. Toskin KD and Pak AN: Use of tube decompression enterography in the diagnosis of acute obstruction of the small intestine. Klin Khir 2: 22-24, 1988 (In Russian)

7. Yao HW, Fu W, Wang DC, Yuan J, Zhang TL and Xiu DR: Long naso-intestinal tube decompression versus octreotide in the treatment of early post-operative inflammatory ileus. Zhonghua Wai Ke Za Zhi 48: 564-568, 2010 (In Chinese).

8. Li de C, Li RH and Tian Q: Efficacy of intestinal decompression with long nasointestinal tube and selective contrast radiography in the treatment of small bowel obstruction in elderly patients. Minerva Chir 71: 85-90, 2016.

9. Zhang L, Gong JF, Ni L, Chen QY, Guo Z, Zhu WM, Li N and Li JS: Influence of preoperative nutritional support on surgical outcomes of chronic radiation enteritis patients complicated with intestinal obstruction. Zhonghua Wei Chang Wai Ke Za Zhi 16: 340-344, 2013 (In Chinese). 